\title{
ARQUITECTURA FOLIAR DE DIEZ ESPECIES DE ENCINO (QUERCUS, FAGACEAE) DE MÉXICO
}

\author{
Isaac Said Rodríguez Rivera y Silvia Romero Rangel \\ Universidad Nacional Autónoma de México, Facultad de Estudios Superiores \\ Iztacala, Laboratorio de Ecología y Taxonomía de Árboles y Arbustos, \\ Avenida de los Barrios 1, Los Reyes Iztacala, \\ 54090 Tlalnepantla, Estado de México, México.
}

\section{RESUMEN}

Se hizo el estudio de la arquitectura foliar de diez especies del género Quercus. Se obtuvieron cuatro patrones de venación: el mixto craspedódromo-camptódromo se presentó en $Q$. deserticola, Q. eduardi, Q. frutex y Q. greggii; el mixto craspedódromoeucamptódromo en $Q$. obtusata, $Q$. potosina y $Q$. scytophylla; el broquidodrómo en $Q$. elliptica y el camptódromo en $Q$. repanda y $Q$. splendens. En todos los taxa se observaron cinco niveles de venación; nueve presentan areolas cuadrangulares y sólo $Q$. repanda las tiene pentagonales. Los estomas sólo se observaron en el envés de las láminas de la hoja. Las especies de la sección Quercus los mostraron de tipo anomocítico y las de la sección Lobatae son ciclocíticos. Los índices estomáticos obtenidos fueron de 36 a 88 en la sección Quercus y de 19 a 42 en la sección Lobatae.

Palabras clave: estomas, Fagaceae, Quercus, venación foliar.

\begin{abstract}
The study of the leaf architecture of ten species of the genus Quercus was made. Four types of venation were observed: the mixed craspedodromous-camptodromous in $Q$. deserticola, Q. eduardi, Q. frutex and Q. greggii; mixed craspedodromous-eucamptodromous in the species $Q$. obtusata, Q. potosina and $Q$. scytophylla; brochidodromous in $Q$. elliptica, and camptodromous in Q. repanda and Q. splendens. All the taxa have five orders of venation; nine species show quadrangular areoles and only $Q$. repanda shows pentagonal areoles. The species of the section Quercus have anomocitic stomates, while those of the section Lobatae have cyclocitic stomates. Stomates are present only on abaxial surface of the leaf. The stomatal indices obtained ranged from 36 to 88 in the section Quercus and from 19 to 42 in the section Lobatae.
\end{abstract}


Key words: Fagaceae, leaf venation pattern, Quercus, stomates.

\section{INTRODUCCIÓN}

El género Quercus, el más grande de la familia Fagaceae, incluye alrededor de 500 especies en todo el mundo. Nixon (1993) reconoce para este taxon dos subgéneros: Quercus y Cyclobalanopsis; al subgénero Quercus lo divide en tres secciones: Lobatae, Protobalanus y Quercus. En México están representadas estas tres últimas.

Se desconoce el número exacto de especies de Quercus en México; Trelease (1924) incluye 253, Miranda y Hernández-X. (1963) 250, Martínez (1954) reconoce 350, Rzedowski (1978) estima que podría haber 150 a 200 y Nixon (1993) dice que existen de 135 a 150, de las cuales más de la mitad (86) son endémicas.

Respecto a su distribución, se conocen encinares en todos los estados de la República, con excepción de Yucatán y Quintana Roo, y se localizan desde el nivel del mar hasta los 3100 m s.n.m. (Rzedowski, 1978).

La importancia de los bosques de Quercus en México radica principalmente en el hecho de que junto con los miembros del género Pinus constituyen la mayor parte de la cubierta vegetal de áreas de clima templado y semihúmedo; sin embargo no se limitan a estas condiciones, pues penetran en regiones de clima caliente formando también bosques, no faltan en las francamente húmedas, son elementos del bosque tropical perennifolio y bosque mesófilo de montaña, y aún existen en las zonas semiáridas siendo parte del matorral xerófilo, asumiendo con frecuencia forma arbustiva (Rzedowski, 1978). Además, por los usos actuales que se dan a la madera (leña y carbón) y los potenciales (obtención de celulosa, forraje, medicinal, reforestación y ornato), hacen del género Quercus uno de mayor importancia económica (Zavala, 1995).

El género es considerado taxonómicamente complicado, debido a la gran variabilidad morfológica relacionada con factores ambientales, daños ocasionados a las ramas y la edad de los individuos; tal circunstancia ha provocado confusiones en la delimitación de las especies, haciéndose necesario el estudio de las poblaciones en su hábitat. La búsqueda de más caracteres diagnósticos, sobre todo en los aspectos de arquitectura foliar y morfología de las estructuras reproductoras (Romero et al., 2002) muy probablemente contribuirá a afinar los límites taxonómicos. Aunque se ha invocado a la hibridación como causa de variabilidad morfológica de los en- 
cinos, ésta no siempre es fácil de identificar en el caso del grupo estudiado y será necesario documentarla con trabajos de biología molecular.

La descripción detallada de caracteres foliares, será de gran ayuda en trabajos taxonómicos y probablemente permitirá discernir tendencias filogenéticas y conocer los efectos del ambiente en la morfología de las hojas (Hickey, 1974).

Hickey (1974) utiliza el término arquitectura foliar para designar la ubicación y la forma de aquellos elementos que constituyen la expresión externa de la estructura de la hoja, incluyendo tipo de venación, configuración marginal, forma de la hoja, posición y tipo de tricomas, así como de estomas. A este respecto se ha encontrado que la mayoría de las familias y géneros poseen modelos consistentes de arquitectura foliar.

En la mayoría de las hojas la venación está claramente diferenciada en clases. Las venas de una clase particular desarrollan un curso y un modelo propio distinto de los demás. Esto hace que el reconocimiento de órdenes de venación sea esencial al describir la arquitectura foliar. La descripción de las hojas no sólo se basa en caracteres cualitativos, sino también en los cuantitativos, como la relación largo/ancho de la lámina, los ángulos formados entre la vena primaria y las secundarias y los ángulos establecidos por la base y el ápice (Hickey et al., 1999).

Actualmente, existe un número importante de estudios de arquitectura foliar de angiospermas; entre ellos se encuentran los de Conover (1983), Cuerrier et al. (1990), Foster (1950), Franck (1976), Galndolfo y Romero (1992), Hershkovitz (1991), Hickey (1973), Hickey y Taylor (1989), Levin (1986), Lis (1992), Pole (1991), Pray (1954, 1959), Ramji (1961), Rury (1976) y Todzia y Keating (1991). Sin embargo, son pocos los que se han realizado sobre Fagaceae; Manos (1993) se basa en el estudio de los tricomas para reconocer cinco especies de la sección Protobalanus y propone una hipótesis de variación geográfica e hibridación entre pares de especies de este grupo. Por su parte Llamas et al. (1995) reconocen, por medio de características de pelos, ocho especies de encinos en la Península Ibérica y con base en estos caracteres definen una clave para la identificación de las mismas.

Valencia y Delgado (1995) utilizan la arquitectura foliar como una herramienta taxonómica para reconocer individuos de Quercus affinis y Q. laurina. Romero (2000) describe y compara los patrones de venación, junto con otros caracteres, de diez especies de la serie Acutifoliae. Martínez (2002) da cuenta de los patrones de venación y el tipo de dientes presentes en Quercus sartorii y Q. xalapensis. 
El objetivo de este trabajo es describir la arquitectura foliar de diez especies mexicanas de Quercus, pertenecientes a las secciones Lobatae y Quercus, así como determinar el tipo de estomas y el índice estomático, con la finalidad de confirmar la utilidad taxonómica de la arquitectura foliar, por lo que se eligieron taxa morfológicamente bien definidos.

\section{MÉTODOS}

Se estudiaron los ejemplares de diez especies de encino depositados en los herbarios de la Facultad de Estudios Superiores Iztacala de la Universidad Nacional Autónoma de México (IZTA), del Instituto de Biología de la misma universidad (MEXU) y de la Escuela Nacional de Ciencias Biológicas del Instituto Politécnico Nacional (ENCB).

Se tomaron muestras de 124 ejemplares de los diez taxa, abarcando su distribución geográfica, las mismas que se diafanizaron y tiñeron para describir la arquitectura foliar, determinar la ubicación, el tipo de estomas y calcular el índice estomático.

Para realizar la diafanización y tinción, las hojas seleccionadas se sometieron a un proceso de aclaramiento, para lo cual se sumergieron en una solución de hidróxido de sodio $(\mathrm{NaOH})$ a $5 \%$ y se dejaron hervir durante 10 minutos, después se lavaron en agua corriente y se colocaron en una solución caliente de hipoclorito de sodio $(\mathrm{NaClO})$ a $30 \%$ hasta que quedaron blancas, enseguida se lavaron con agua corriente. Para realizar la tinción, las hojas aclaradas se colocaron en safranina alcohólica a 1\% durante 45 minutos. Después se hicieron cambios graduales de alcohol, a $60 \%$, 70\%, 80\% y 96\%, de 15 minutos cada uno; posteriormente se colocaron en xilol hasta lograr su aclaramiento. Las muestras obtenidas se montaron entre dos cristales con resina para microscopía. Las muestras diafanizadas se depositaron en el Laboratorio de Ecología y Taxonomía de Árboles y Arbustos de la Facultad de Estudios Superiores Iztacala.

Se obtuvieron imágenes de la arquitectura foliar con un digitalizador Alpha Innotech y con una cámara Nikon FX-35DX adaptada a los microscopios estereoscópico y óptico.

La descripción de la arquitectura foliar de cada especie se realizó de acuerdo con Hickey (1974), adicionando los caracteres de tricomas, mismos que se describieron siguiendo a Jones (1986). Se determinaron los tipos de estomas (Dilcher, 1974) y se calculó el índice estomático: 


$$
\mathrm{IE}=\frac{\mathrm{NE}}{\mathrm{NE}+\mathrm{CE}} \times 100
$$

donde, NE es el número de estomas por campo de observación a 100x y CE es el número de células epidérmicas por campo de observación a 100x.

Se tomaron fotografías y se hicieron dibujos de la morfología de cada taxon.

\section{RESULTADOS}

Descripciones de la arquitectura foliar

Quercus deserticola Trel. Mem. Nat. Acad. Sci. 20: 79, t. 113. 1924.

Hojas maduras de forma elíptica, elíptica-ancha o elíptica-angosta; ápice obtuso convexo, pocas veces redondeado; base simétrica, redondeada, a veces cordada; margen serrado a crenado, revoluto, 2-4 dientes (raramente 5) por lado, con mucrones rectos de 0.3-0.5 mm de largo; venación mixta craspedódromacamptódroma; relación ancho de lámina/diámetro de vena primaria: fuerte; venas secundarias que divergen de la vena primaria en ángulo agudo-moderado (45$65^{\circ}$ ), venas secundarias superiores que divergen en ángulos más agudos que las inferiores, con 2-4 venas intersecundarias; venas terciarias con ángulo de origen agudo-agudo o agudo-recto, percurrentes alternas; venas de orden cuarto de tamaño moderado y trayectoria ortogonal; venas de orden quinto de tamaño fino o moderado y trayectoria ortogonal; vena última marginal incompleta; vénulas ramificadas una vez; areolas bien desarrolladas, con disposición ordenada, de forma cuadrangular y tamaño pequeño; haz verde lustroso con tricomas fasciculados de 7-11 ramas, distribuidos uniformemente; envés pálido con abundantes tricomas fasciculados de 7-9 ramas, con estípite largo, además con tricomas glandulares de color amarillo; los tricomas en el envés forman un tomento delgado amarillento; epidermis del envés poco ampulosa y papilosa; pecíolo de 3-6 mm de largo y 0.91.5 mm de diámetro; estomas de tipo anomocítico (Fig. 1).

Reconocimiento: Quercus deserticola se distingue por sus venas secundarias superiores que divergen en ángulo más agudo que las inferiores y tricomas fasciculados con estípite largo en el haz de la lámina foliar.

Ejemplares examinados: Distrito Federal. Santa Fe, Martínez 56 (ENCB)

Estado de México. Mpio. Tepotzotlán: Sierra de Alcaparrosa, Reynoso 1662 (IZTA). Mpio. San Andrés Timilpan: Sierra de San Andrés, Camacho \& Santiago 


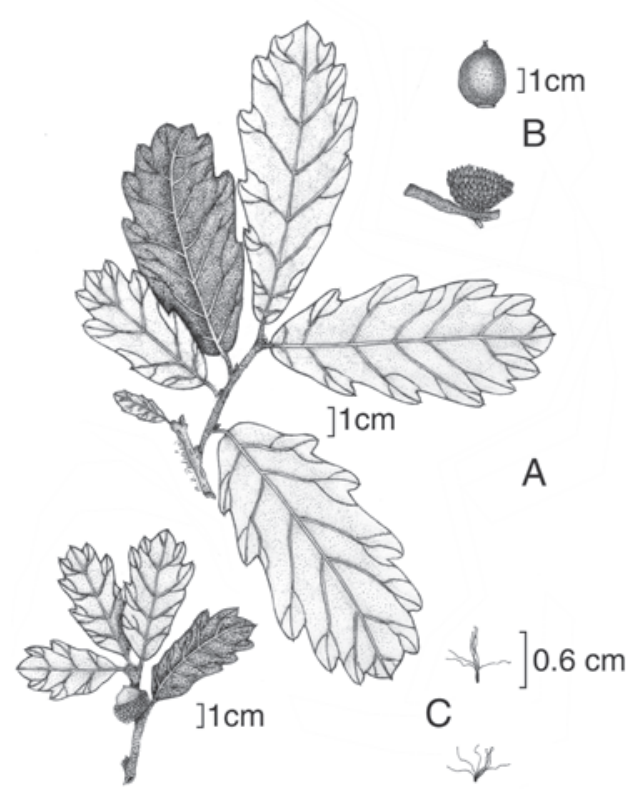

$1 \mathrm{~cm}-$
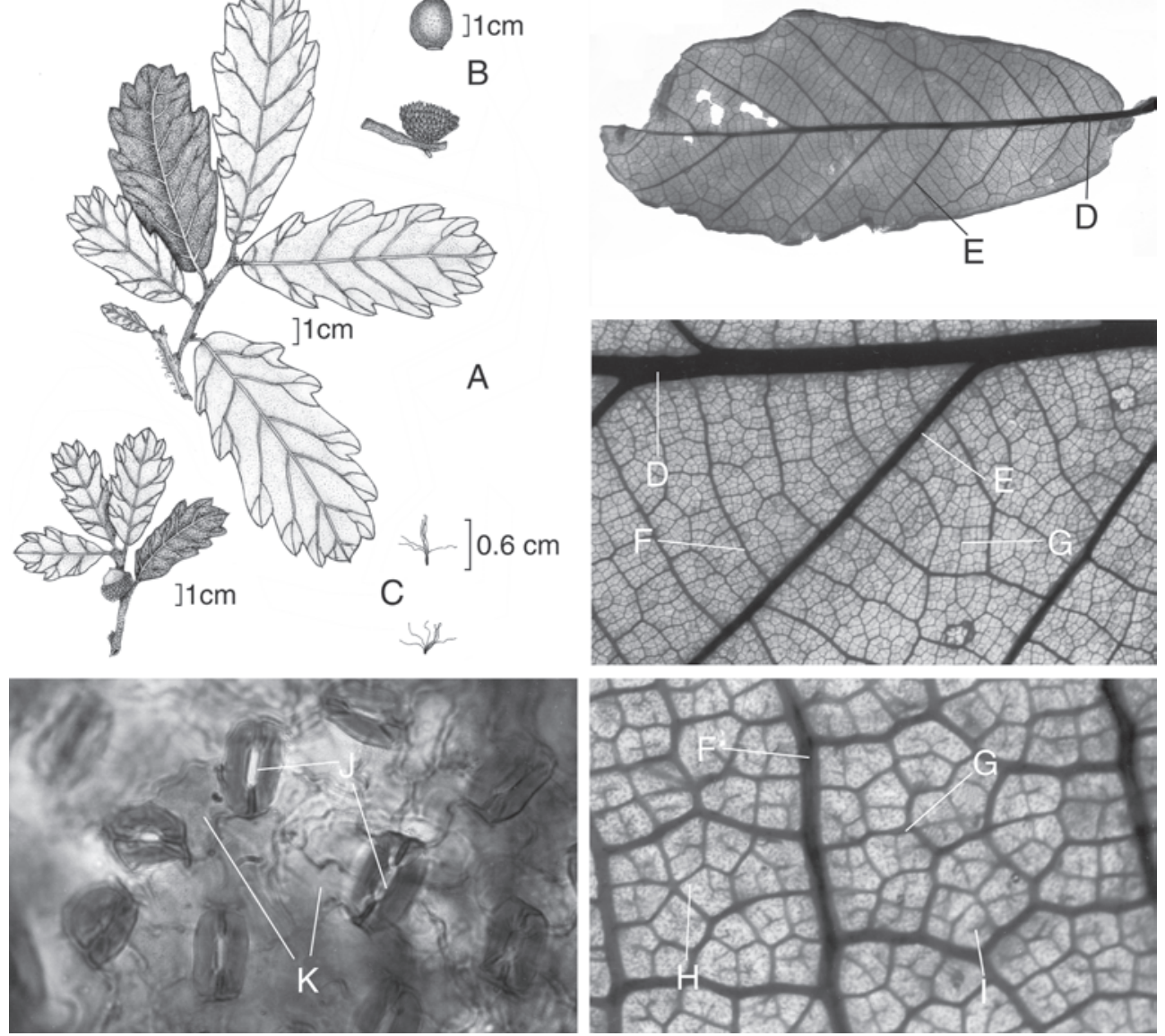

Fig. 1. Quercus deserticola. Morfología general: A. rama con hojas; B. fruto y cúpula; C. tricomas (Díaz 2406, ENCB). Niveles de venación: D. vena primaria (1ª); E. vena secundaria $\left(2^{a}\right)$; F. vena terciaria $\left(3^{a}\right)$; G. vena de orden cuarto $\left(4^{a}\right)$; H. vena de quinto orden $\left(5^{\mathrm{a}}\right)$; I. vénulas; estoma de tipo anomocítico: J. estoma; K. células epidérmicas (Reynoso 1663, IZTA).

379 (IZTA). Mpio. Tepotzotlán: Sierra de Alcaparrosa, Reynoso 1663 (IZTA). Mpio. Tepotzotlán: Sierra de Alcaparrosa, Rzedowski 29940 (ENCB). Jalisco. Mpio. Atamejac de Brizuela: El Márquez, González 2223 (ENCB). Mpio. Zapotlanejo: Cerro de Santa Fe, González 2134 (ENCB). Mpio. Jocotepec: Sierra de Tecuan, González 679 (ENCB). Mpio. Jocotepec: Sierra de Tecuan, V. de Puga 673 (ENCB). Michoacán. Mpio. Coeneo: 2 km al NE de Bellas Fuentes, Díaz 2406 (ENCB). Mpio. La Piedad: 
Cerro Grande de Cujaruato, Rzedowski \& McVaugh 500 (ENCB). Oaxaca. Mpio. Ocotlán de Morelos: La Garzona, Robles 42 (ENCB).

Quercus eduardi Trel. Mem. Nat. Acad. Sci. 20: 121, t. 219. 1924.

Hojas maduras de forma elíptica o elíptica-angosta; ápice obtuso-convexo; base simétrica, cordada; margen serrado, 3-5 dientes por lado, de borde plano o revoluto, frecuentemente grueso, con aristas de 0.5-1.2 mm de largo; venación mixta craspedódroma-camptódroma; relación ancho de lámina/diámetro de vena primaria: fuerte; venas secundarias que divergen de la vena primaria en ángulo agudo-moderado $\left(45-65^{\circ}\right)$ o agudo-angosto $\left(<45^{\circ}\right)$, presenta de $2-4$ venas intersecundarias; venas terciarias con ángulo de origen agudo-agudo o agudo-recto, percurrentes alternas; venas de cuarto orden de tamaño moderado y trayectoria ortogonal; venas de quinto orden de tamaño moderado o fino y trayectoria ortogonal; vena última marginal incompleta; vénulas ramificadas una vez; areolas bien desarrolladas, orientadas, de forma cuadrangular y tamaño mediano; haz verde lustroso con tricomas fasciculados contortos, sésiles, con 14-17 ramas; envés con tricomas fasciculados contortos, con 14-17 ramas, sésiles, distribuidos uniformemente, axilas con tricomas fasciculados extendidos, de 14-17 ramas y estípite largo, en ocasiones con tricomas glandulares de color amarillo; epidermis lisa; pecíolo de 3-8 mm de largo y 0.6-1.2 mm de diámetro; estomas de tipo ciclocítico (Fig. 2).

Reconocimiento: Quercus eduardi se distingue por sus areolas de tamaño mediano y tricomas fasciculados del envés contortos.

Ejemplares examinados: Durango. Mpio. Súchil: La Corraleña, Acevedo 500 (ENCB). Mpio. Durango: $15 \mathrm{~km}$ al S de la ciudad de Durango, Spellenberg \& González 10261 (ENCB). Guanajuato. Mpio. Juventino Rosas: 23.5 km al NW de Juventino Rosas, Galván 3950 (IZTA). Mpio. Dolores Hidalgo: 9 km al E-NE de Santa Rosa, Galván 4486 (ENCB). Mpio. Guanajuato: Mesa de los HernándezPeña Colorada, Mares \& Estrada 135 (ENCB). Mpio. Guanajuato: 5 km al E-NE de Guanajuato, Galván 3546 (ENCB). Mpio. Guanajuato: Cañada de la Virgen, Cerro de las Peñas, Martínez C. 622 (IZTA). Mpio. Juventino Rosas: 23.5 km al N-NW de Juventino Rosas, Galván 3950 (IZTA). Hidalgo. Mpio. Metztitlán: 2 km al NW de Zoquizoquipan, Martínez 771 (IZTA). Mpio. Tulancingo: 4 km al W de Tulancingo, Hernández 3315 (ENCB). Jalisco. Mpio. Ahualulco de Mercado: La Estanzuela, González \& Mancinas 611 (ENCB). Mpio. Villa Guerrero: 30 km al N de Bolaños, Luquin, Ornelas \& Santana 177 (ENCB). Querétaro. Mpio. Te- 

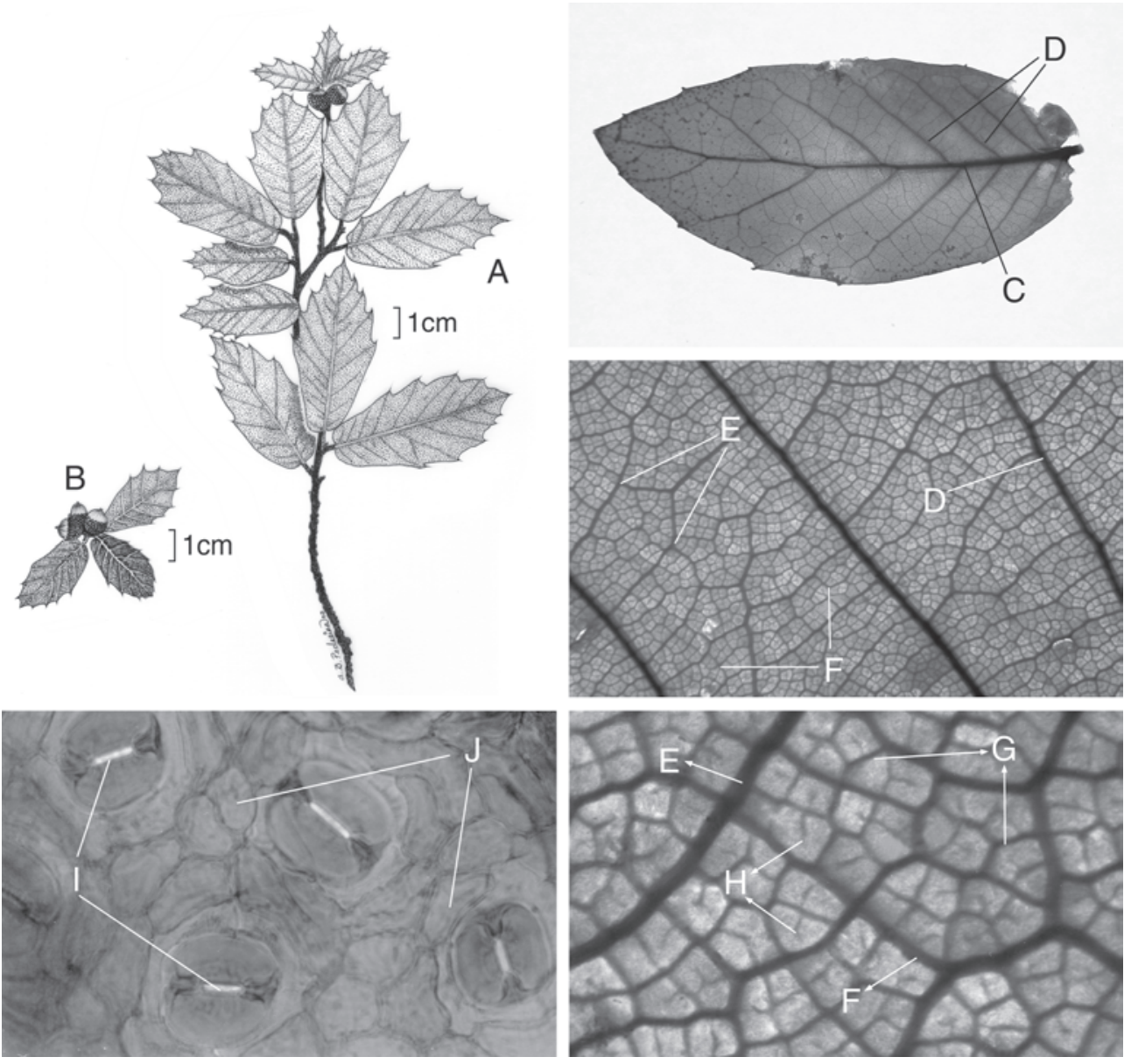

Fig. 2. Quercus eduardi. Morfología general: A. rama con hojas (Martínez C. 622, IZTA); B. hojas con fruto (Galván 3950, IZTA). Niveles de venación: C. vena primaria (1ª); D. vena secundaria $\left(2^{\mathrm{a}}\right)$; E. vena terciaria $\left(3^{\mathrm{a}}\right)$; F. vena de cuarto orden $\left(4^{\mathrm{a}}\right)$; $\mathrm{G}$. vena de quinto orden (5); H. vénulas; estoma de tipo ciclocítico: I. estoma; J. células epidérmicas (Martínez C. 622, IZTA).

quisquiapan: Sierra de Mastranzo, Paray 2482 (ENCB). San Luis Potosí. Mpio. Mexquitic de Carmona: Rincón del Hormiguero, Hernández (ENCB).

Quercus elliptica Née. Anales Ci. Nat. 3: 278. 1801.

Hojas maduras coriáceas de forma elíptica, elíptica-angosta o elíptica-ancha; ápice obtuso a redondeado; base cordada; margen entero, borde poco revoluto, no presenta aristas ni mucrones; venación broquidódroma; relación ancho de lámina/ 

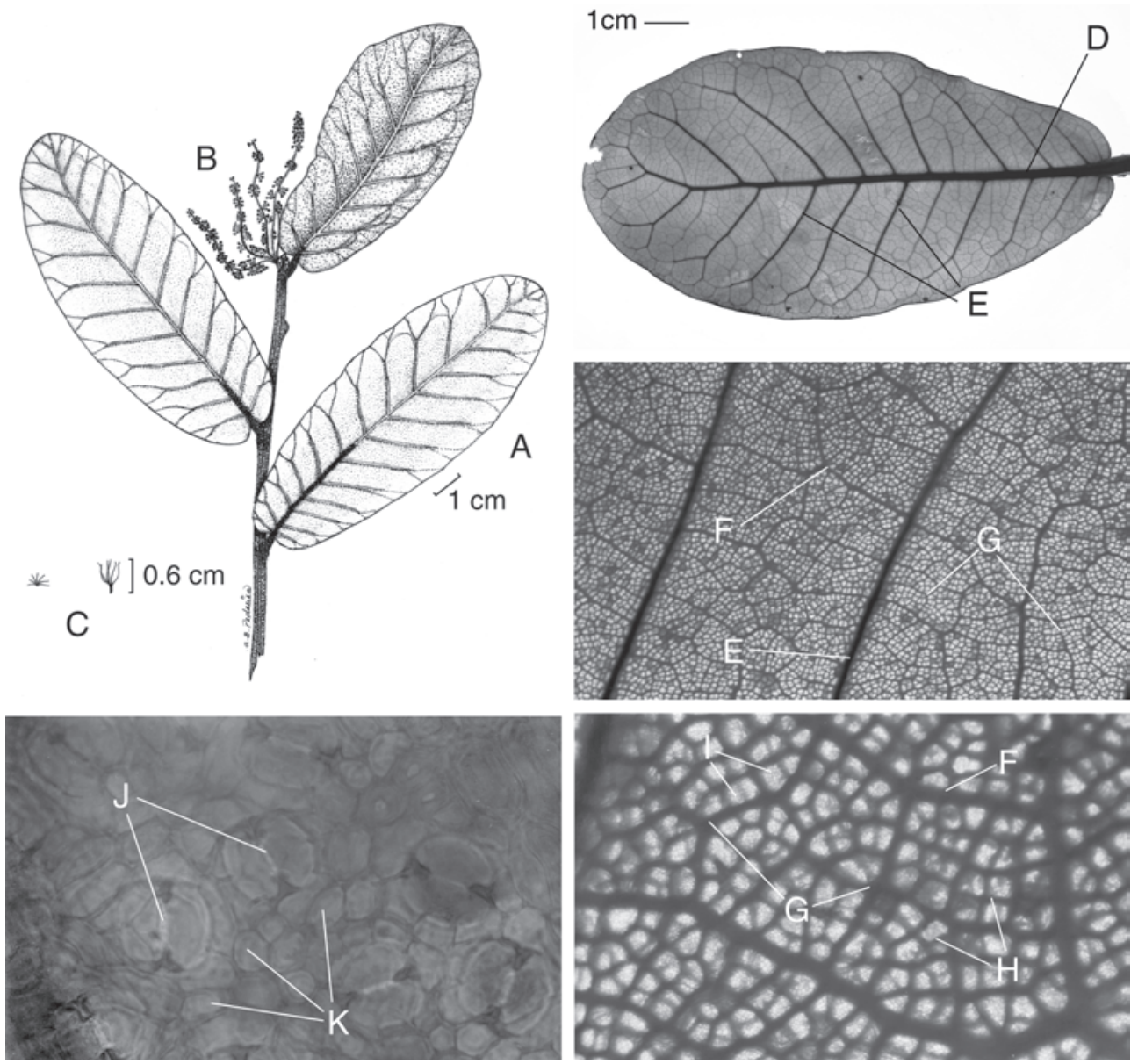

Fig. 3. Quercus elliptica. Morfología general: A. rama con hojas; B. inflorescencia; C. tricomas (Rojas \& Romero 3956, IZTA). Niveles de venación: D. vena primaria (1ª); E. vena secundaria ( $\left.2^{a}\right)$; F. vena terciaria $\left(3^{a}\right)$; G. vena de cuarto orden $\left(4^{a}\right)$; H. vena de quinto orden $\left(5^{a}\right)$; I. vénulas; estoma de tipo anomocítico: J. estoma; K. células epidérmicas (Rojas \& Romero 3956, IZTA).

diámetro de vena primaria: fuerte; venas secundarias que divergen de la vena primaria en un ángulo agudo-moderado $\left(45^{\circ}-65^{\circ}\right)$, en pocas ocasiones mayor, presenta 10-20 venas intersecundarias; venas terciarias con ángulo de origen agudo-recto o agudo-agudo, percurrentes alternas; venas de cuarto orden de tamaño moderado y trayectoria al azar; venas de quinto orden de tamaño grueso y trayectoria ortogonal; vena última marginal formando arcos, en ocasiones incompleta; vénulas simples; 
areolas bien desarrolladas, con disposición al azar, forma cuadrangular y tamaño pequeño; haz verde pálido con tricomas fasciculados de 5-7 ramas, estípite corto; envés glabro, a veces con pelos estrellados de 5-7 ramas, con estípite largo, principalmente en las axilas y vena primaria; epidermis generalmente lisa; pecíolo de 3-5 mm de largo y de 1.5-3 mm de diámetro; estomas de tipo anomocítico (Fig. 3).

Reconocimiento: Quercus elliptica se distingue por el margen entero de las láminas foliares y por presentar gran cantidad de venas intersecundarias (10-20).

Ejemplares examinados: Estado de México. Mpio. Tejupilco: km 23 El Corupo-Nanchititla, Rojas \& Romero 3956 (IZTA). Mpio. Tejupilco: 12 km El Corupo-Nanchititla, Rojas \& Romero S.I.D. (IZTA). Mpio. Tejupilco: La Joyita de Cuajinicuiles, Abundiz 664 (IZTA). Guerrero. Mpio. San Miguel Totolapan: Agua Vicente, Diego 2878 (ENCB). Mpio. San Miguel Totolapan: Cerro de la Vaca, Rodríguez 31 (ENCB). Jalisco. Mpio. Mascota: 25 km al NW de Mascota, González 1127 (ENCB). Mpio. Puerto Vallarta: 3 km al N de Saucillo, González 1953 (ENCB). Mpio. Talpa: km 17 brecha Zimapan-Cuale, González 1096 (ENCB). Nayarit. Mpio. El Nayar: 3 km al N de Santa Cruz de Guaybel, González 3347 (ENCB). Oaxaca. Mpio. Santos Reyes Tepejillo: a 16 km de Santiago Juxtlahuaca, Calzada 21918 (MEXU). Mpio. San Juan Mixtepec: 6 km al E de Independencia, Salas 206 (ENCB). Mpio. San Juan Mixtepec: 5 km al E de San Juan Mixtepec, Salas 77 (ENCB). Mpio. San Juan Ñumi: km 3 San Juan Mixtepec-Santo Domingo Yosoñama, Sánchez 8 (ENCB). Mpio. Santiago Juxtlahuaca: 5-6 km de El Manzanar, Calzada 21743 (MEXU). Sinaloa. Mpio. Concordia; Potrerillos, Vega 1975 (ENCB).

Quercus frutex Trel. Mem. Nat. Acad. Sci. 20: 82, t. 120. 1924.

Hojas maduras coriáceas, de forma elíptica o elíptica-angosta; lámina simétrica; ápice obtuso convexo, redondeado o pocas veces agudo; base simétrica redondeada o cordada; margen dentado-crenado o entero, borde revoluto, 2-4 dientes por lado cuando los presenta; dientes con mucrones convexos a redondeados, de 0.2-0.3 mm de largo; venación mixta craspedódroma-camptódroma; relación ancho de lámina/diámetro de vena primaria: fuerte; venas secundarias que divergen de la vena primaria en ángulo agudo moderado (45-65) y en ocasiones más de $65^{\circ}$ sin pasar de $80^{\circ}$, presenta 2-5 venas intersecundarias; venas terciarias con ángulo de origen agudo-agudo, percurrentes alternas, recorrido derecho; venas de cuarto orden de tamaño moderado y trayectoria ortogonal; venas de quinto orden de tamaño fino, moderado o grueso, trayectoria ortogonal; vena última marginal incompleta; vénulas ramificadas una vez; areolas bien desarrolladas, con disposición ordenada, 

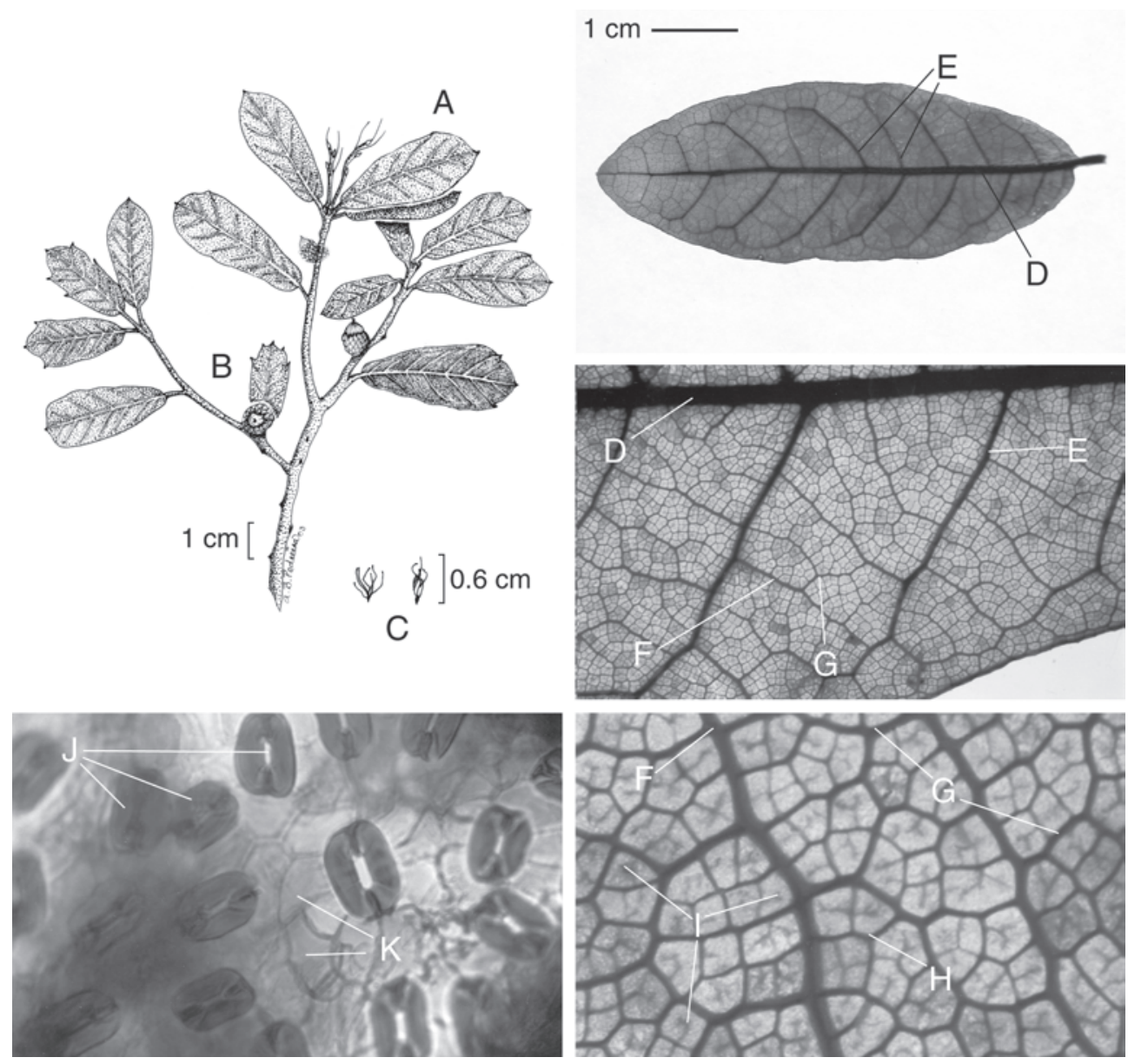

Fig. 4. Quercus frutex. Morfología general: A. rama con hojas; B. frutos; C. tricomas (Román 622, IZTA). Niveles de venación: D. vena primaria (1 $\left.{ }^{\mathrm{a}}\right)$; E. vena secundaria $\left(2^{\mathrm{a}}\right)$; F. vena terciaria ( $\left.3^{a}\right)$; G. vena de cuarto orden $\left(4^{a}\right)$; H. vena de quinto orden ( $\left.5^{a}\right)$; I. vénulas; estoma de tipo anomocítico: J. estoma; K. células epidérmicas (Román 622, IZTA).

forma cuadrangular y tamaño pequeño; haz verde con tricomas fasciculados de 6-8 ramas, sésiles; envés amarillento tomentoso, tricomas fasciculados de 8 ramas, con estípite largo; epidermis ampulosa o poco ampulosa, papilosa; pecíolo de 2-5 mm de largo y 0.6-1.5 mm de diámetro; estomas de tipo anomocítico (Fig. 4).

Reconocimiento: Quercus frutex se distingue porque sus venas secundarias divergen de la primaria en ángulo agudo moderado $\left(45^{\circ}-65^{\circ}\right)$ y en ocasiones de más de $65^{\circ}$. 
Ejemplares examinados: Estado de México. Mpio. Chicoluapan: $5 \mathrm{~km}$ al E de Coatepec, Román 622 (IZTA). Mpio. Texcoco: Cerro Tetzcutzingo, Pulido 466 (ENCB). Mpio. Huehuetoca: Cerro Sincoque, Rojas \& Romero 700 (IZTA). Mpio. Tepotzotlán: Cerro de la Cruz, Rzedowski 37055 (ENCB). Mpio Texcoco: 15 km al NE de Texсосо, Brizuela 273 (ENCB). Hidalgo. Mpio. El Oro de Hidalgo: Basoco, Rojas \& Romero 3327 (IZTA). Mpio. Zempoala: San Mateo Tlacomulco, Ventura 3718 (ENCB). Mpio. Zempoala: Sierra de los Pitos, Rzedowski 27072 (ENCB). Querétaro. Mpio. Amealco: $12 \mathrm{~km}$ al E de Coroneo, Puig 6037 (ENCB).

Quercus greggii (DC.) Trel. Mem. Nat. Acad. Sci. 20: 78, t. 109. 1924.

Hojas maduras coriáceas, algunas cóncavas, de forma elíptica o elíptica-ancha; ápice obtuso convexo; base simétrica, obtusa, cordada; margen marcadamente revoluto, dentado-crenado de 2-3 dientes por lado, pocas veces entero, mucrones de forma recta de 0.2-0.5 mm de largo; venación pinnada mixta craspedódromacamptódroma; relación ancho de lámina/diámetro de vena primaria: fuerte a masiva; venas secundarias que divergen de la vena primaria en ángulo agudo moderado $\left(45^{\circ}-65^{\circ}\right)$, y en ocasiones agudo-angosto $\left(>45^{\circ}\right)$, presentan $2-3$ venas intersecundarias simples; venas terciarias con ángulo de origen agudo-agudo, percurrentes alternas y recorrido derecho; venas de cuarto orden de tamaño fino, trayectoria al azar; venas de quinto orden de tamaño grueso y trayectoria ortogonal; vena última marginal incompleta; vénulas simples; areolas bien desarrolladas, con disposición al azar, de forma irregular y tamaño pequeño; haz verde-grisáceo a café, con las venas muy marcadas, presenta tricomas fasciculados de 15 ramas sésiles; envés pardo, tomento delgado, tricomas fasciculados de 15 ramas, sésiles o con estípite corto; epidermis ampulosa y papilosa con tricomas glandulares de color ámbar; pecíolo de 3-7 mm de largo y 1.2-1.8 mm de diámetro; estomas de tipo anomocítico (Fig. 5).

Reconocimiento: Quercus greggii se distingue porque el margen de la lámina foliar es marcadamente revoluto y sus areolas son de tamaño pequeño.

Ejemplares examinados: Coahuila. Mpio. Arteaga: Cerro de la Viga, Passini y Robert 6638 (ENCB). Hidalgo. Mpio. Epazoyucan: 1 km al W de Nopalillo, Rzedowski 37217 (ENCB). Mpio. Zempoala: Sierra de los Pitos, Rzedowski 31189 (ENCB). Mpio. Zempoala: Sierra de los Pitos, Benítez 320 (ENCB). Mpio. Epazoyucan: Cerro Grande, Rzedowski 28955 (ENCB). Mpio. Zempoala: Sierra de los Pitos, Benítez 46 (ENCB). Nuevo León. Mpio. Galeana: Cerro del Potosí, Gutiérrez 2213 (ENCB). Mpio. Santiago: Sierra Rancho Nuevo, Valdez 741 (ENCB). Mpio. 

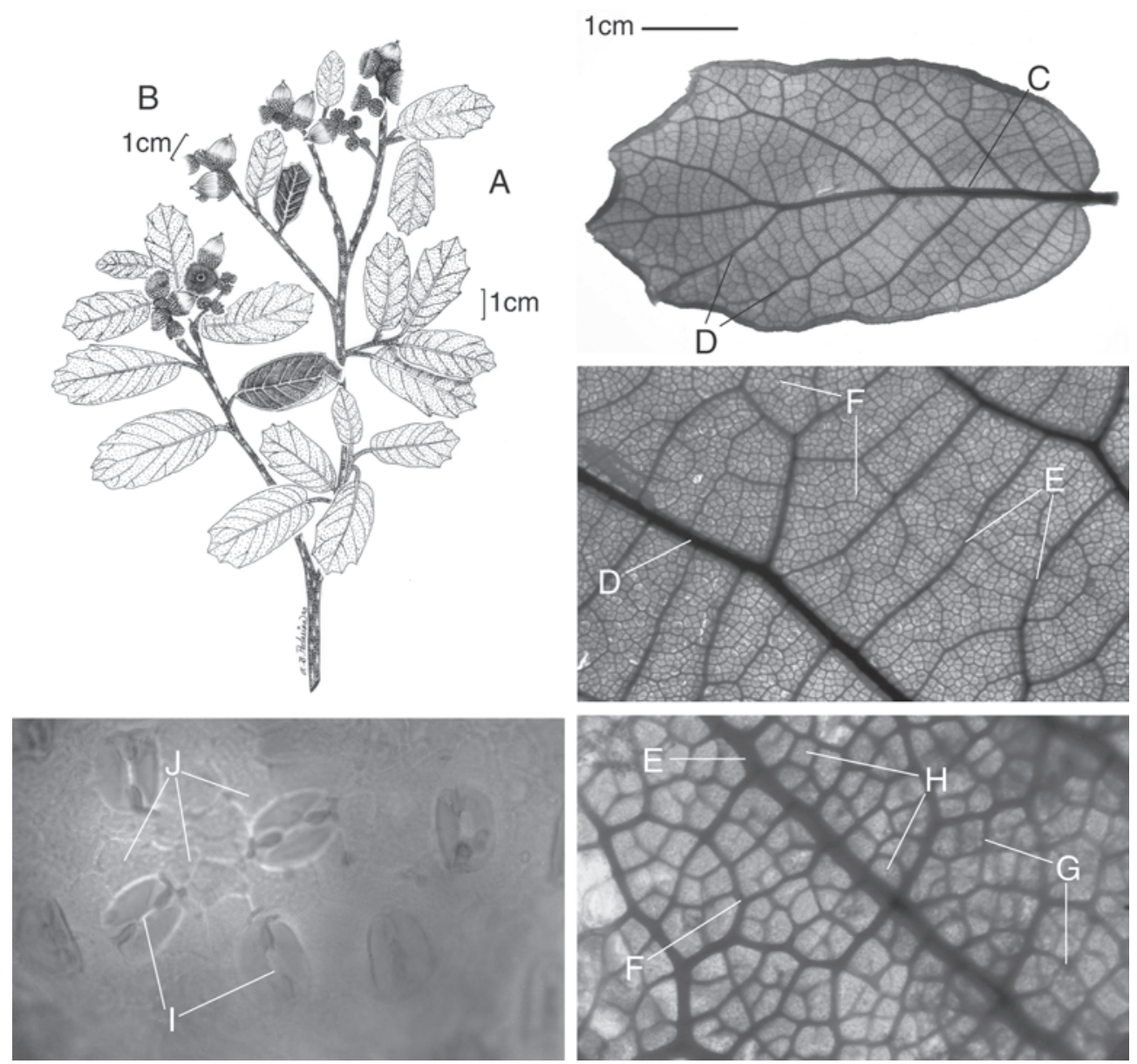

Fig. 5. Quercus greggii. Morfología general: A. rama con hojas (Hinton et al. 17442, ENCB); B. frutos (Benítez 46, ENCB). Niveles de venación: C. vena primaria (1a); D. vena secundaria ( $\left.2^{\mathrm{a}}\right)$; E. vena terciaria ( $3^{\mathrm{a}}$ ); F. vena de cuarto orden $\left(4^{\mathrm{a}}\right)$; G. vena de quinto orden ( $\left.5^{\mathrm{a}}\right)$; H. vénulas; estoma de tipo anomocítico: I. estoma; J. células epidérmicas (Rzedowski 7877, ENCB).

Zaragoza: San Antonio-Peña Nevada, Hinton et al. 17442 (ENCB). Mpio. Galeana: Camino al Cerro Potosí, Dorr \& Atkins 2306 (ENCB). Puebla. Mpio. Acajete: cerro Pinal al SE de la Malinche, Ern 215 (ENCB). Querétaro. Mpio. Cadereyta: Sierra Peña Azul, Zamudio 3039 (ENCB). San Luis Potosí. Mpio. Cerro de San Pedro: cerros al S de Jesús María, Rzedowski 7877 (ENCB). Mpio. Catorce: Sierra de Catorce, Wendt, Lott, García \& Rebolledo 2230 (ENCB). 
Quercus obtusata Humb. \& Bonpl. Pl. Aequinoct. 2: 26, t. 76. 1809.

Hojas maduras de forma obovada-angosta, obovada-ancha o elíptica; ápice obtuso convexo o redondeado; base simétrica, obtusa redondeada o cordada; margen dentado-crenado, borde poco revoluto o revoluto, de 3-8 dientes por lado, provistos de mucrones convexos o extendidos de 0.3-0.4 mm de largo; venación pinnada, mixta craspedódroma-eucamptódroma; relación ancho de lámina/diámetro de vena primaria: moderada o fuerte; venas secundarias que divergen de la vena primaria en ángulo agudo-moderado $\left(45^{\circ}-65^{\circ}\right)$ o agudo-angosto $\left(<45^{\circ}\right)$, con 2-4 venas intersecundarias; venas terciarias con ángulo de origen agudo-agudo, percurrentes alternas y de recorrido derecho; venas de cuarto orden de tamaño moderado y trayectoria ortogonal; venas de quinto orden de tamaño fino y trayectoria ortogonal; vena última marginal formando arcos o incompleta; vénulas ramificadas una vez; areolas bien desarrolladas, con disposición al azar, de forma cuadrangular y tamaño mediano; haz verde lustroso con tricomas fasciculados de 6-8 ramas sésiles; envés con tomento verde amarillento, tricomas fasciculados de 8 ramas, sésiles o con estípite corto además de tricomas glandulares de color ámbar o rojo; epidermis papilosa, ampulosa o poco ampulosa; pecíolo de 6-12 mm de largo y 1-1.6 mm de diámetro; estomas de tipo anomocítico (Fig. 6).

Reconocimiento: Quercus obtusata se distingue por presentar generalmente láminas foliares de forma obovada.

Ejemplares examinados: Distrito Federal. Cañada de Contreras, Paray 598 (ENCB). Estado de México. Mpio. Acambay: cerro Hordingo, Estrada 1443 (IZTA). km 16 camino Sultepec-San Miguel Totolmoloya, Torres 195 (IZTA). Mpio. Tenango del Aire: Rancho San Luis Aculco, Hinton et al. 17989 (ENCB). Guanajuato. Mpio. Dolores Hidalgo: 7 km al NE de Santa Rosa, Galván 3985 (ENCB). Guerrero. Mpio. Taxco de Alarcón: Puerto de Cucharillo, Valencia 71 (ENCB). Hidalgo. Mpio. Nicolás Flores: Cerro Bando, Rangel 182 (ENCB). Jalisco. Mpio. Talpa de Allende: cerro Don Pedro, González 3268 (ENCB). Michoacán. Mpio. Quiroga: cerro La Acumara, Díaz 1048 (ENCB). Mpio. Pátzcuaro: Cerro del Estribo, Díaz 1094 (ENCB). Querétaro. Mpio. Amealco: Camino entre Amealco y Santiago, Argüelles 2942 (IZTA). Mpio. San Joaquín: Otatal a $10 \mathrm{~km}$ de San Joaquín, Huerta $X-723$ (ENCB).

Quercus potosina Trel. Mem. Nat. Acad. Sci. 20: 84, pl. 123. 1924.

Hojas maduras de forma elíptica-ancha u obovadas; ápice obtuso o convexo; base asimétrica, obtusa a cordada; margen dentado o crenado, pocas veces entero, borde no revoluto, con 1 a 6 dientes por lado, provistos de mucrones convexos o 

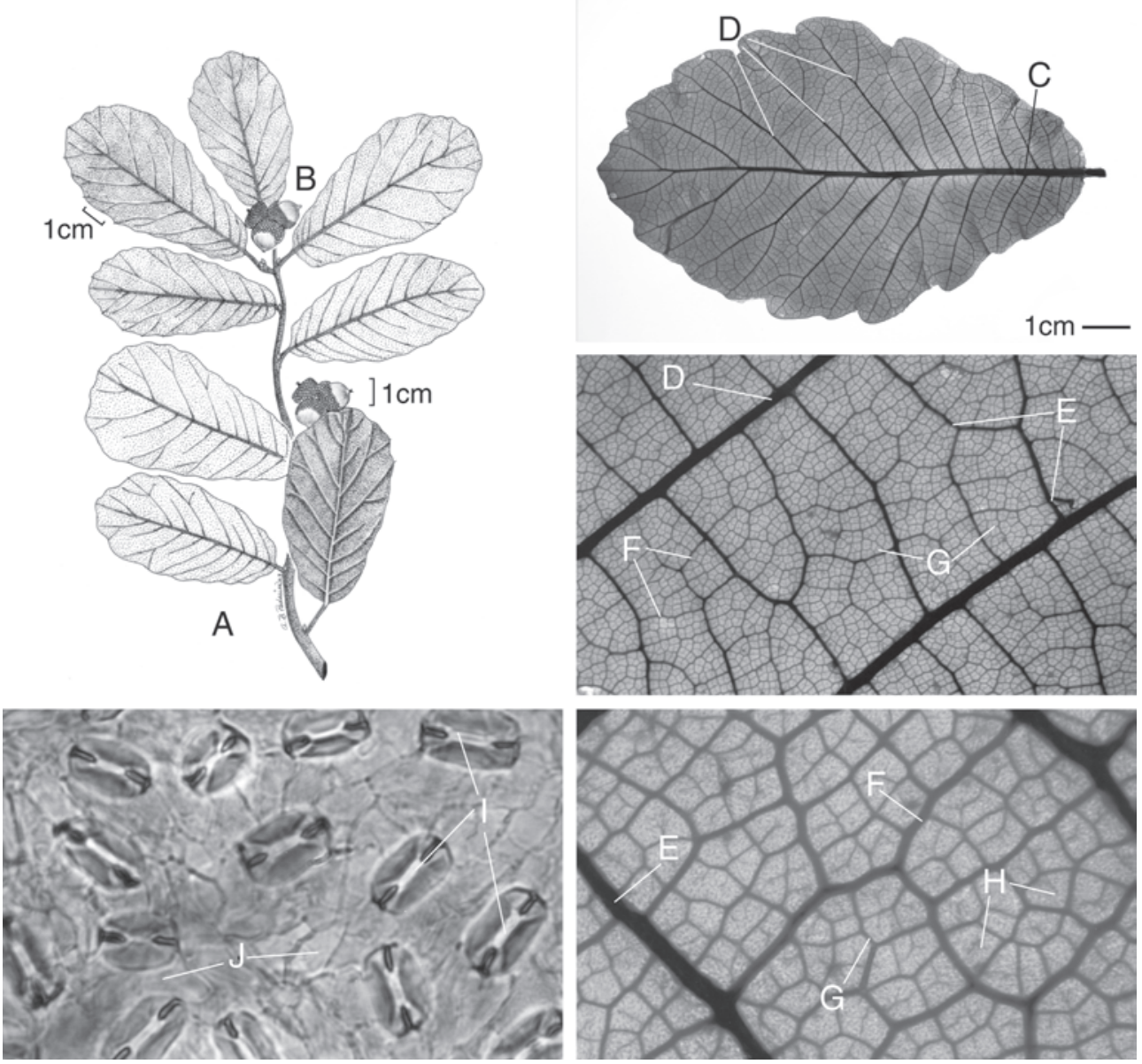

Fig. 6. Quercus obtusata. Morfología general: A. ramas con hojas (Galván 3985, ENCB); B. frutos (Díaz 1094, ENCB). Niveles de venación: C. vena primaria (1ª); D. vena secundaria (2ª); E. vena terciaria $\left(3^{\mathrm{a}}\right)$; F. vena de cuarto orden $\left(4^{\mathrm{a}}\right)$; G. vena de quinto orden $\left(5^{\mathrm{a}}\right)$; H. vénulas; estoma de tipo anomocítico: I. estoma; J. células epidérmicas (Díaz 1048, ENCB).

rectos de 0.2-0.5 mm de largo; venación pinnada mixta, craspedódroma-eucamptódroma; relación ancho de lámina/diámetro de vena primaria: débil o moderada; venas secundarias que divergen de la vena primaria en ángulo agudo-moderado (45-65), presenta 2-6 venas intersecundarias simples; venas terciarias con ángulo de origen agudo-agudo, percurrentes alternas y recorrido derecho; venas de cuarto orden de tamaño fino o moderado y trayectoria ortogonal; venas de quinto orden de tamaño moderado y trayectoria ortogonal; vena última marginal incompleta; vénu- 
las ramificadas una vez; areolas bien desarrolladas con disposición al azar de forma cuadrangular y tamaño pequeño; haz un tanto lustroso, verde oscuro o verde-amarillento, con tricomas fasciculados de 6-8 ramas, sésiles; envés pubescente, con pelos estrellados de 6-8 ramas, sésiles, y tricomas glandulares de color ámbar; epidermis ampulosa o poco ampulosa, papilosa; pecíolo de 2-5 mm de largo por 0.8-1.5 mm de diámetro; estomas de tipo anomocítico (Fig. 7).

Reconocimiento: Quercus potosina se distingue por su relación ancho de lámina/diámetro de vena primaria: débil o moderada.

Ejemplares examinados: Durango. Mpio. Súchil: arroyo Pito Real, Alvarado 273 (ENCB). Mpio. Súchil: Sierra de Urica, González \& Acevedo 3627 (ENCB). Estado de México. Mpio. Villa Nicolás Romero: La Colmena, Quintero 28 (IZTA). Guanajuato. Mpio. Ocampo: 4 km al S de Santa Bárbara, Carranza 4323 (IZTA). Hidalgo. Mpio. Zempoala: Sierra de los Pitos, Benítez 38 (ENCB). Mpio. Epazoyucan: Cerro Grande 2 km al S de Epazoyucan, Rzedowski 28963 (ENCB). Mpio. Epazoyucan: Cerro Grande 2 km al NW de Epazoyucan, Rzedowski 32051 (ENCB). Jalisco. Mpio. Ojuelos de Jalisco: 1 km al NW de Los Papás de Arriba, Santana \& Arreola 1528 (ENCB). Mpio. Lagos de Moreno: 3 km al S de El Paso, González 1389 (ENCB). Querétaro. Mpio. Tolimán: en Gudiños, Zamudio 2570 (ENCB).

Quercus repanda Humb. \& Bonpl. Plant. Aequinoct. 2:31-32, t. 79. 1809.

Hojas maduras de forma elíptica u obovada-angosta; ápice obtuso convexo, pocas veces redondeado; base simétrica o asimétrica, obtusa redondeada; margen dentado crenado o entero, revoluto o poco revoluto, con mucrones rollizos o convexos de 0.2-0.4 cm de largo; venación camptódroma; relación ancho de lámina/diámetro de vena primaria: fuerte; venas secundarias que divergen de la primaria en ángulo agudo-moderado ( $\left.45-65^{\circ}\right)$ y en ocasiones agudo-angosto $\left(<45^{\circ}\right)$, presenta $2-4$ venas intersecundarias simples; venas terciarias con ángulo de origen agudo-agudo o agudo-recto, percurrentes alternas, recorrido convexo; venas de cuarto orden de tamaño fino o moderado, de trayectoria ortogonal; venas de quinto orden de tamaño fino y trayectoria ortogonal; vena última marginal incompleta; vénulas simples; areolas bien desarrolladas, orientadas, forma irregular o pentagonal, tamaño pequeño; haz verde grisáceo con tricomas fasciculados sésiles de 14-16 ramas; envés densamente tomentoso, pardo-amarillento con tricomas fasciculados de 16-21 ramas, sésiles o con estípite corto, algunas veces presenta tricomas glandulares de color ámbar; epidermis ampulosa y papilosa; pecíolo de 3-7 mm de largo por 1-1.2 mm de diámetro; estomas de tipo anomocítico (Fig. 8). 

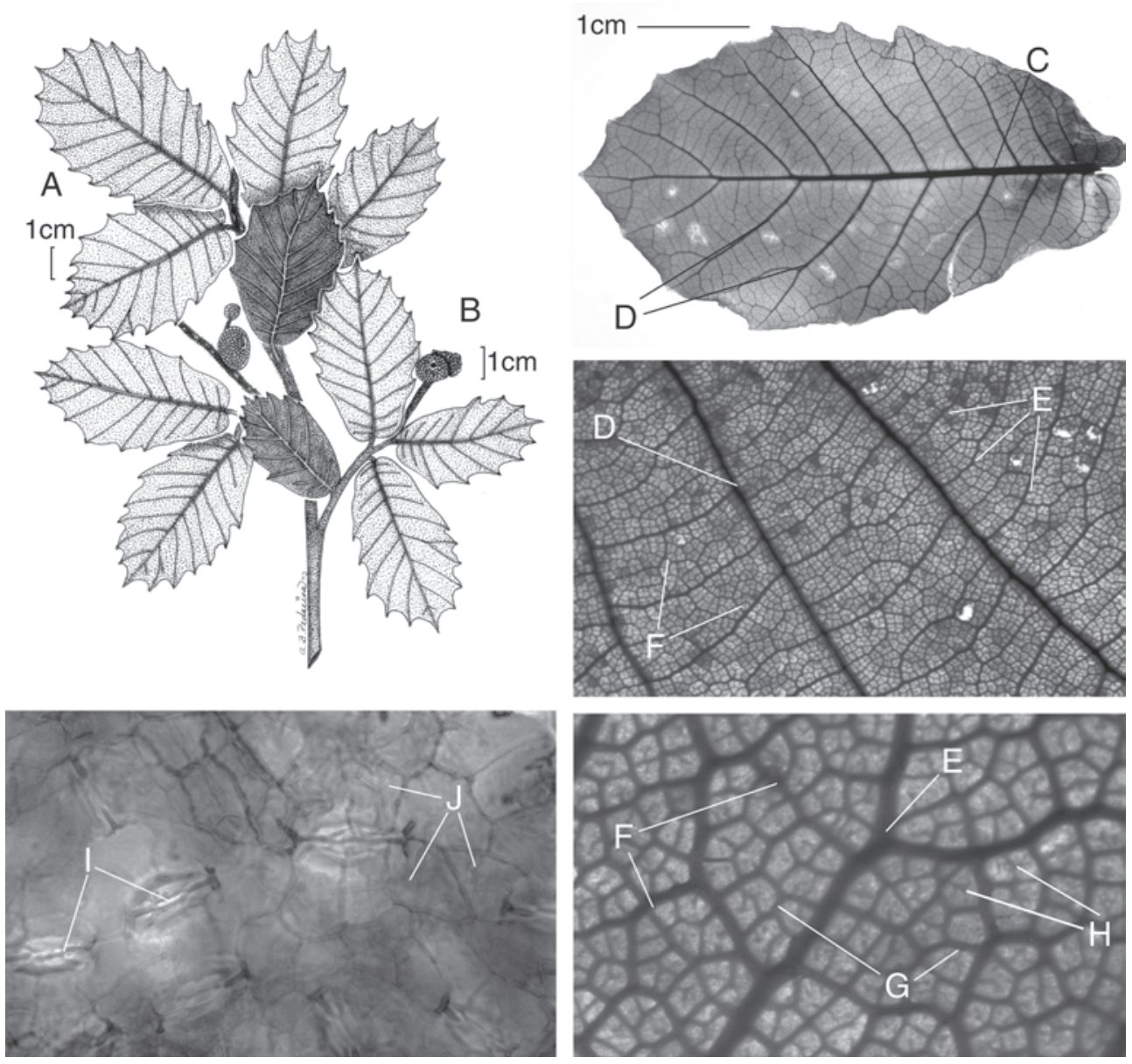

Fig. 7. Quercus potosina. Morfología general: A. rama con hojas; B. frutos (Santana \& Arreola 1528, ENCB). Niveles de venación: C. vena primaria $\left(1^{\mathrm{a}}\right)$; D. vena secundaria $\left(2^{\mathrm{a}}\right)$; E. vena terciaria ( $\left.3^{a}\right)$; F. vena de cuarto orden $\left(4^{a}\right)$; G. vena de quinto orden $\left(5^{a}\right)$; H. vénulas; estoma de tipo anomocítico: I. estoma; J. células epidérmicas (Carranza 4323, IZTA).

Reconocimiento: Quercus repanda se distingue porque la última venación marginal es incompleta y porque sus tricomas fasciculados del envés tienen 16 a 21 ramas.

Ejemplares examinados: Estado de México. Mpio. Villa Nicolás Romero: $5 \mathrm{~km}$ al E de Santiago Tlazala, Rzedowski 27117 (ENCB). Hidalgo. Mpio. Agua Blanca de Iturbide, Turra 2948 (ENCB). Mpio. Real del Monte: Peñas Largas cerca 

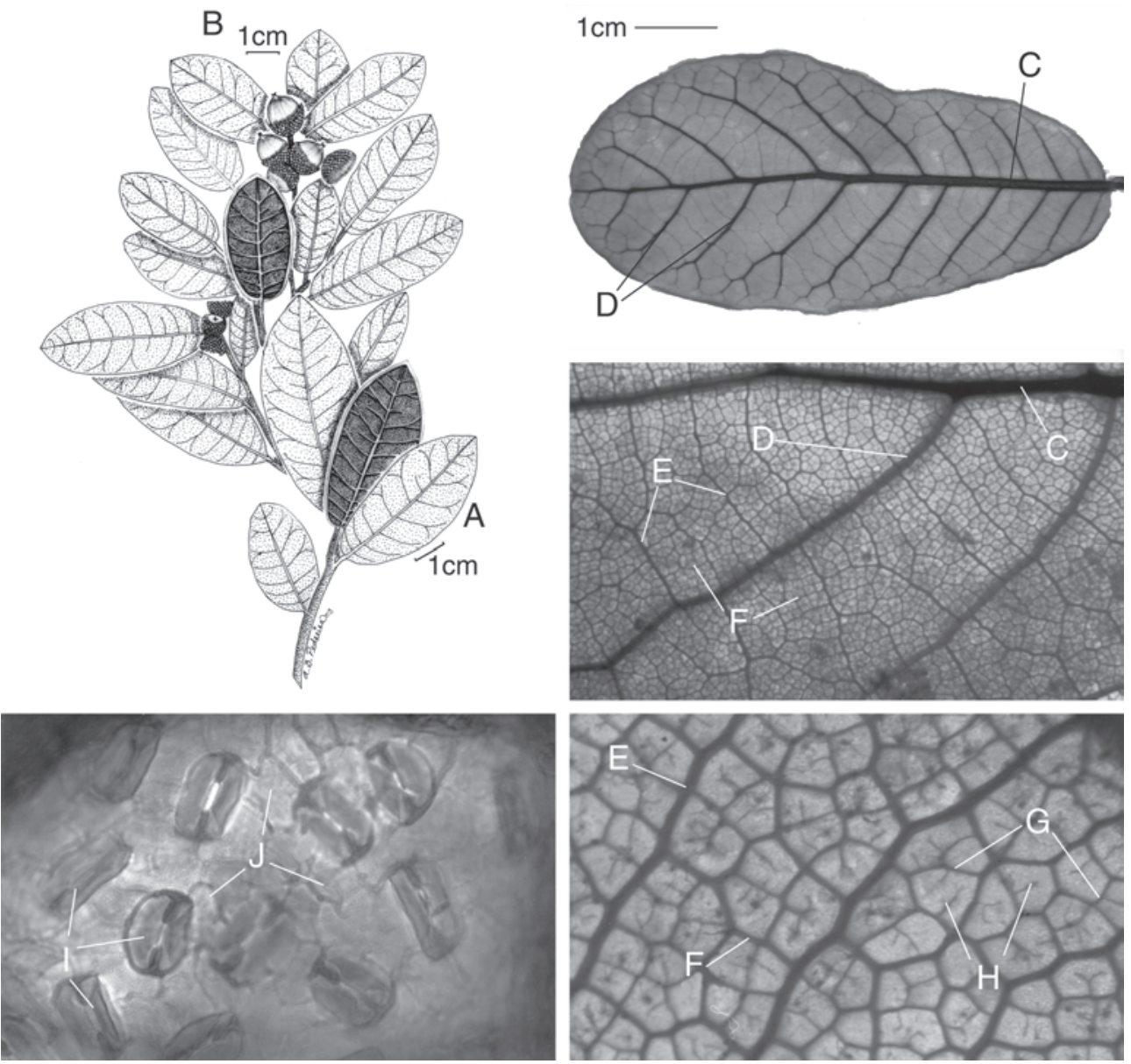

Fig. 8. Quercus repanda. Morfología general: A. rama con hojas (Vela 710, ENCB); B. frutos (Rzedowski 22172, ENCB). Niveles de venación: C. vena primaria (1ª); D. vena secundaria $\left(2^{\mathrm{a}}\right)$; E. vena terciaria $\left(3^{\mathrm{a}}\right)$; F. vena de cuarto orden $\left(4^{\mathrm{a}}\right)$; G. vena de quinto orden $\left(5^{\mathrm{a}}\right)$; H. vénulas; estoma de tipo anomocítico: I. estoma; J. células epidérmicas (Medina \& Barrios 3020, ENCB).

de Tezoantla, Rzedowski 22172 (ENCB). Mpio. Epazoyucan: $4.5 \mathrm{~km}$ al E de Real del Monte, González 958 (ENCB). Mpio. Tepeapulco: cerro Xihuingo, Rzedowski 31421 (ENCB). Cerca de Tezuantla, Espinoza 407 (ENCB). Querétaro. Mpio. San Joaquín: Campo Alegre 1 km al S de San Joaquín, Medina \& Barrios 3020 (ENCB). Mpio. Pinal de Amoles: Cerro de la Calentura, Rzedowski 48239 (ENCB). San Luis Potosí. Mpio. San Luis Potosí: Cerro del Órgano al SW de San Luis Potosí, Rzedowski 8488 (ENCB). Mpio. Villa de Reyes: Las Ciénegas, Robert 1183 (ENCB). 
Mpio. Guadalcázar: Cerro Grande al NW de Guadalcázar, Rzedowski 5980 (ENCB). Veracruz. Mpio. Huayacocotla: Jarillos, límite de Veracruz e Hidalgo, Nevling \& Goméz-Pompa 1760 (ENCB). Huayacocotla, Vela 710 (ENCB).

Quercus scytophylla Liebm. Overs. Kongel. Danske Vidensk. Selsk. Forth. Medlemmers Arbeider p. 180. 1854.

Hojas maduras de forma obovada-angosta, obovada-ancha o elíptica; ápice agudo; base simétrica, aguda, rara vez obtuso-redondeada; margen dentado-serrado, borde revoluto; 3-7 dientes por lado, presenta aristas de 0.5-2.5 cm de largo; venación pinnada, mixta craspedódroma-eucamptódroma; relación ancho de lámina/diámetro de vena primaria: moderada o fuerte; venas secundarias que divergen de la vena primaria en ángulo agudo-moderado $\left(45-65^{\circ}\right)$ o agudo-angosto $\left(<45^{\circ}\right)$, presenta de 3-12 venas intersecundarias simples; venas terciarias con ángulo de origen agudo-agudo, percurrentes alternas, de recorrido convexo; venas de cuarto orden de tamaño fino o moderado, de trayectoria ortogonal; venas de quinto orden de tamaño fino a grueso, de trayectoria ortogonal; vena última marginal incompleta; vénulas simples, algunas veces ramificadas una vez; areolas bien desarrolladas, con disposición orientada, de forma cuadrangular y tamaño pequeño; haz verde-grisáceo, glabro, en ocasiones presenta tricomas fasciculados de 14 ramas en la base; envés con tomento denso color blanco, tricomas fasciculados sésiles de 14-16 ramas, en ocasiones presenta tricomas glandulares de color ámbar; epidermis ampulosa y papilosa; pecíolo de 7-24 mm de largo y de 1.3-2 mm de diámetro; estomas de tipo ciclocítico (Fig. 9).

Reconocimiento: Quercus scytophylla se distingue por la base aguda de sus láminas foliares y el indumento blanco del envés.

Ejemplares examinados: Estado de México. Mpio. Tejupilco: Parque cinegético Nanchichitla, Abundiz 565 (IZTA). Mpio. Temascaltepec: km 30 de la carretera federal 130, cercanías de Mesón Viejo, Orozco 49b (IZTA). Guerrero. Mpio. Leonardo Bravo: km 19 de la desv. Puerto del Gallo-Filo de Caballo, Valencia 441 (ENCB). Mpio. Leonardo Bravo: 23 km SW Filo de Caballo, Valencia 440 (ENCB). Mpio. Tixtla: cerro Xomislo, Cobián 11 (ENCB). Mpio. Tixtla de Guerrero: carretera Azoquipa-Duraznos, 2 km de las desviación, Valencia 150 (ENCB). Jalisco. Mpio. Tecatitlán: Martínez 182 (ENCB). Mpio. Talpa de Allende: cerro Don Pedro, González y col. 3269 (ENCB). Mpio. Talpa de Allende: montañas 11-12 mi. al S de Talpa, McVaugh 21423 (ENCB). Michoacán. Mpio. Hidalgo: El Caracol, Unidad Industrial Forestal "San José” 2466 (ENCB). Mpio. Aguililla: Cerritos de Agua, McVaugh 17838 (ENCB). Mpio. Aguililla: pie de montañas al NW de Aguililla, 

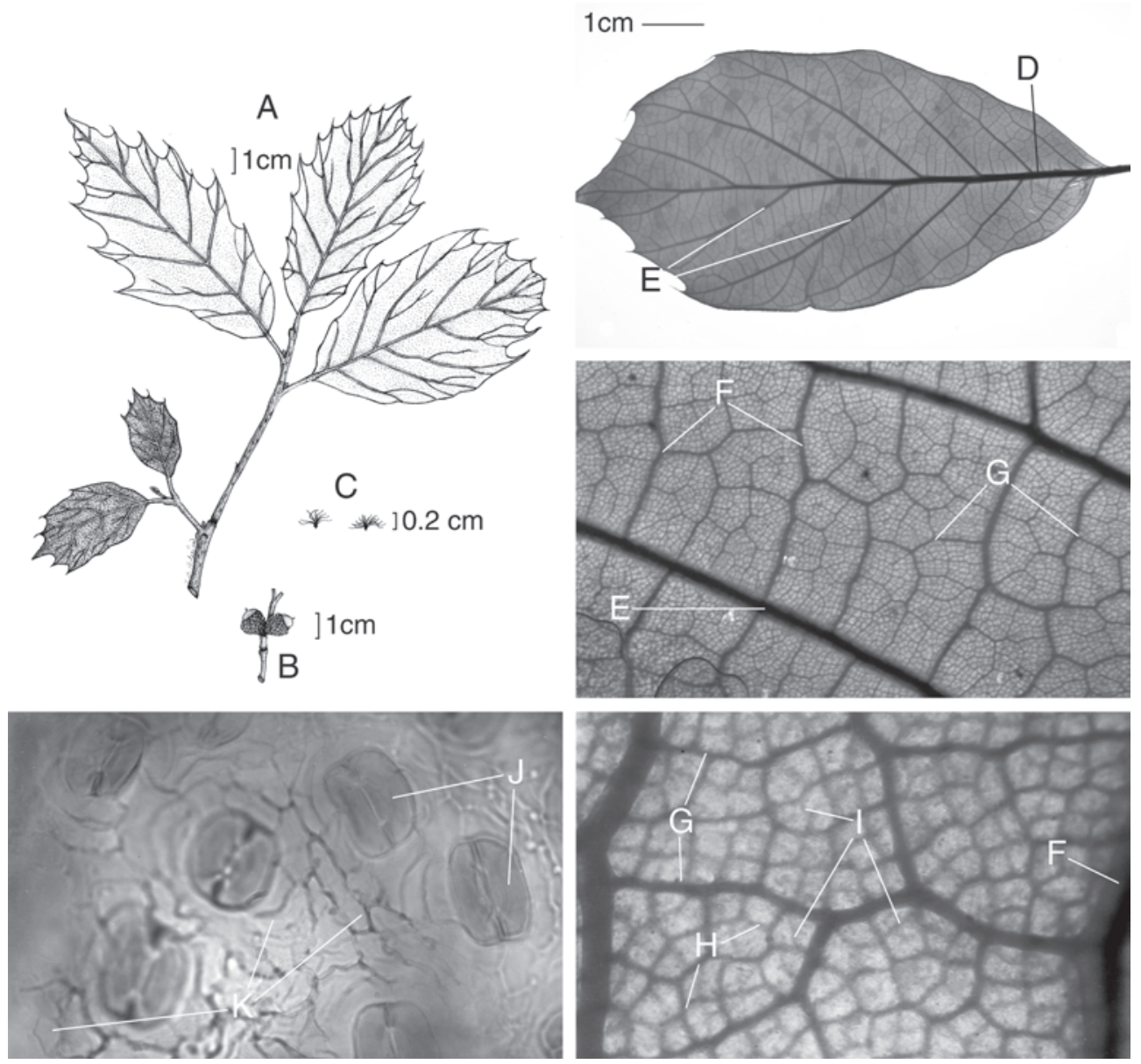

Fig. 9. Quercus scytophylla. Morfología general: A. rama con hojas; B. frutos; C. tricomas (Orozco 49b, IZTA). Niveles de venación: D. vena primaria $\left(1^{\mathrm{a}}\right)$; E. vena secundaria $\left(2^{\mathrm{a}}\right)$; F. vena terciaria $\left(3^{\mathrm{a}}\right)$; G. vena de cuarto orden $\left(4^{\mathrm{a}}\right)$; H. vena de quinto orden $\left(5^{\mathrm{a}}\right)$; I. vénulas; estoma de tipo ciclocítico: J. estoma; K. células epidérmicas (Calzada 21882, IZTA).

McVaugh 22671 (ENCB). Mpio. Aguililla: montañas al NW de Aguililla, McVaugh 22689 (ENCB). Oaxaca. Mpio. San Sebastián Tecomaxtlahuaca: carretera Guadalupe Nundala a 11.6 km de San Sebastián, Calzada 21882 (MEXU). Mpio. Villa Sola de Vega: 15 km al S de Sola de Vega, López 801 (ENCB). Puebla. Mpio. Xicotepec: Necaxa, Vela 506 (ENCB). 
Quercus splendens Née. Anales Ci. Nat. 3: 275. 1801.

Hojas maduras de forma elíptica o elíptica-angosta; ápice obtuso, redondeado o convexo; base simétrica o asimétrica, obtuso-redondeada a veces cordada; margen entero, pocas veces crenado, cartilaginoso, plano o ligeramente revoluto, mucrones rollizos convexos de 0.2-0.4 mm de largo; venación pinnada, camptódroma; relación ancho de lámina/diámetro de vena primaria: moderada a fuerte; venas secundarias que divergen de la vena primaria en ángulo agudo-moderado $\left(45-65^{\circ}\right)$, presenta de 2-12 venas intersecundarias simples; venas terciarias con ángulo de origen agudo-agudo o agudo-recto, percurrentes alternas con recorrido derecho; venas de cuarto orden de tamaño grueso $\mathrm{y}$ trayectoria ortogonal; venas de quinto orden de tamaño fino y trayectoria ortogonal; últimas venas formando arcos o fimbriadas; vénulas ramificadas una vez; areolas bien desarrolladas con disposición orientada, forma cuadrangular y tamaño pequeño; haz de color verde oscuro con tonos rojizos; envés verde, glabrescente, tricomas fasciculados persistentes en las axilas de las nervaduras y vena primaria, estrellados de 8 ramas, con estípite largo o corto; epidermis papilosa, no ampulosa; pecíolo de 3-14 mm de largo y 1-1.5 mm de diámetro; estomas de tipo anomocítico (Fig. 10).

Reconocimiento: Quercus splendens se distingue por sus láminas foliares glabrescentes con tricomas fasciculados persistentes en las axilas de las nervaduras y vena primaria, y últimas venas fimbriadas.

Ejemplares examinados: Durango. Mpio. El Salto: Espinazo del Diablo, carretera Durango-Mazatlán, Cházaro \& Rodríguez 5127 (ENCB). Estado de México. Mpio. Sultepec: km 12 de la carretera Sultepec-San Miguel Totolmoloya, Torres 529 (IZTA). Mpio. Tejupilco: cerro Nanchititla, Matuda 31527 (ENCB). Guerrero. Mpio. Tixtla de Guerrero: falda W del cerro El Fresno, Kruse 1813 (ENCB). Jalisco. Mpio. Jilotlán: km 86 Tecalitlán-Jilotlán de Dolores, Pérez 529 (ENCB). Mpio. Amatepec: cercanías de Amatepec, Matuda 29824 (ENCB). Mpio. Chiquistlán: 10 km al N de La Cuesta, Rzedowski 15140 (ENCB). Mpio. San Martín de Bolaños: Las Treinta Vueltas, 10 km al N de El Platanar, Rzedowski 26208 (ENCB). Mpio. Bolaños: Sierra de Bolaños, Villarreal 1935 (ENCB).

Estomas e índice estomático

Se determinaron dos tipos de estomas: anomocítico, el cual se observó en las especies de la sección Quercus, esta modalidad se caracteriza por presentar las células anexas morfológicamente no diferenciadas del resto de las células epidérmi- 

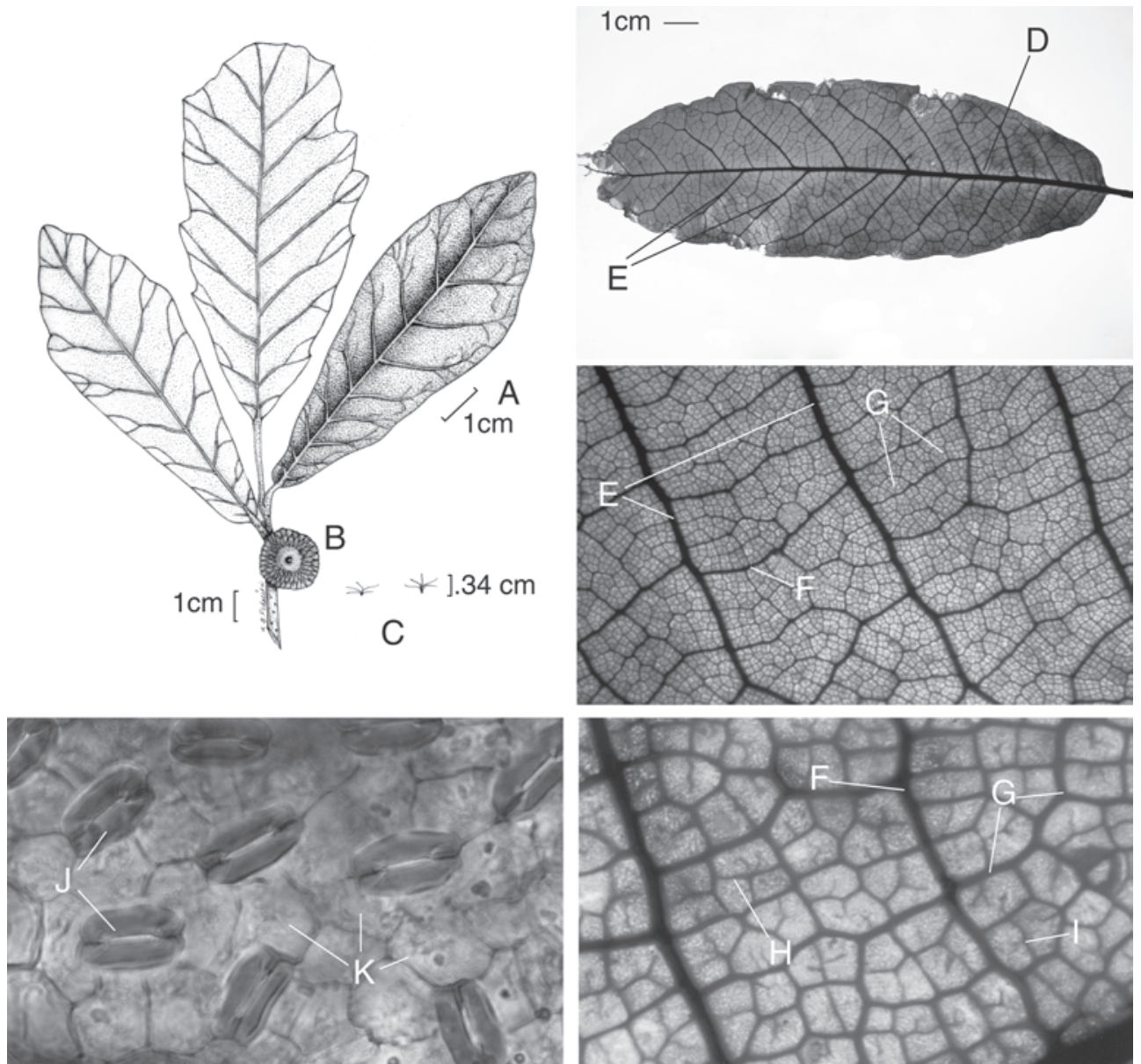

Fig. 10. Quercus splendens. Morfología general: A. rama con hojas; B. fruto; C. tricomas (Torres 529, IZTA). Niveles de venación: D. vena media $\left(1^{\mathrm{a}}\right)$; E. vena secundaria $\left(2^{\mathrm{a}}\right)$; F. vena terciaria $\left(3^{a}\right)$; G. vena de cuarto orden $\left(4^{a}\right)$; H. vena de quinto orden $\left(5^{a}\right)$; I. vénulas; estoma de tipo anomocítico: J. estoma; K. células epidérmicas (Torres 529, IZTA).

cas; y ciclocítico, presente en las especies de la sección Lobatae, el cual se distingue porque sus células anexas son de aspecto diferente del de las epidérmicas normales, formando un anillo de cinco o más células alrededor de las oclusivas (Dilcher, 1974) (Cuadro 1).

El índice estomático (IE) fue muy variable entre los taxa, en las especies de la sección Quercus se observó un intervalo de 36 a 88, mientras que para las de la sección Lobatae fue de 19 a 42, ya que presentan estomas de mayor tamaño (Cuadro 1). 
Cuadro 1. Tipo de estomas e índice estomático (100x).

\begin{tabular}{|c|c|c|c|c|c|}
\hline Especie & Herbario & $\begin{array}{l}\text { Tipo de } \\
\text { estoma }\end{array}$ & $\begin{array}{l}\text { Núm. de } \\
\text { estomas }\end{array}$ & $\begin{array}{c}\text { Núm. de } \\
\text { células } \\
\text { epidérmicas }\end{array}$ & $\begin{array}{c}\text { Índice } \\
\text { estomático } \\
\text { (IE) }\end{array}$ \\
\hline \multicolumn{6}{|l|}{ Q. deserticola } \\
\hline Reynoso 1662 & IZTA & Anomocítico & 22 & 3 & 88 \\
\hline Camacho 379 & IZTA & Anomocítico & 34 & 6 & 85 \\
\hline Reynoso 1663 & IZTA & Anomocítico & 33 & 16 & 67 \\
\hline \multicolumn{6}{|l|}{ Q. eduardi } \\
\hline Martínez 771 & IZTA & Ciclocítico & 12 & 48 & 20 \\
\hline Martínez C. 662 & IZTA & Ciclocítico & 13 & 56 & 19 \\
\hline R \& J Galván 3950 & IZTA & Ciclocítico & 21 & 60 & 25 \\
\hline \multicolumn{6}{|l|}{ Q. elliptica } \\
\hline Calzada 21918 & MEXU & Anomocítico & 18 & 25 & 42 \\
\hline R \& R 3956 & IZTA & Anomocítico & 20 & 54 & 27 \\
\hline R \& R (SID) & IZTA & Anomocítico & 18 & 47 & 28 \\
\hline \multicolumn{6}{|l|}{ Q. frutex } \\
\hline R \& R 3327 & IZTA & Anomocítico & 29 & 15 & 66 \\
\hline Román 622 & IZTA & Anomocítico & 41 & 20 & 67 \\
\hline Puig 6037 & ENCB & Anomocítico & 31 & 24 & 56 \\
\hline \multicolumn{6}{|l|}{ Q. greggii } \\
\hline Zamudio 3039 & ENCB & Anomocítico & 18 & 20 & 47 \\
\hline Rzedowski 7877 & ENCB & Anomocítico & 22 & 30 & 42 \\
\hline Gutiérrez 2213 & ENCB & Anomocítico & 17 & 30 & 36 \\
\hline \multicolumn{6}{|l|}{ Q. obtusata } \\
\hline Estrada 1443 & IZTA & Anomocítico & 28 & 14 & 67 \\
\hline Torres 195 & IZTA & Anomocítico & 24 & 5 & 83 \\
\hline Díaz 1048 & ENCB & Anomocítico & 29 & 10 & 74 \\
\hline \multicolumn{6}{|l|}{ Q. potosina } \\
\hline Carranza 4323 & IZTA & Anomocítico & 18 & 23 & 44 \\
\hline Santana 1528 & ENCB & Anomocítico & 18 & 20 & 47 \\
\hline Alvarado 273 & ENCB & Anomocítico & 19 & 13 & 59 \\
\hline
\end{tabular}


Cuadro 1. Tipo de estomas e índice estomático (100x). Continuación.

\begin{tabular}{|c|c|c|c|c|c|}
\hline Especie & Herbario & $\begin{array}{l}\text { Tipo de } \\
\text { estoma }\end{array}$ & $\begin{array}{l}\text { Núm. de } \\
\text { estomas }\end{array}$ & $\begin{array}{c}\text { Núm. de } \\
\text { células } \\
\text { epidérmicas }\end{array}$ & $\begin{array}{l}\text { Índice } \\
\text { estomático } \\
\text { (IE) }\end{array}$ \\
\hline \multicolumn{6}{|l|}{ Q. repanda } \\
\hline Rzedowski 8488 & ENCB & Anomocítico & 23 & 15 & 61 \\
\hline Nevling 1760 & ENCB & Anomocítico & 27 & 15 & 64 \\
\hline Medina 3020 & ENCB & Anomocítico & 16 & 50 & 24 \\
\hline \multicolumn{6}{|l|}{ Q. scytophylla } \\
\hline Calzada 21882 & MEXU & Ciclocítico & 16 & 28 & 36 \\
\hline Abundiz 565 & IZTA & Ciclocítico & 16 & 23 & 41 \\
\hline Cobián 11 & ENCB & Ciclocítico & 11 & 30 & 27 \\
\hline \multicolumn{6}{|l|}{ Q. splendens } \\
\hline Torres 529 & IZTA & Anomocítico & 22 & 25 & 47 \\
\hline Pérez 985 & ENCB & Anomocítico & 21 & 22 & 49 \\
\hline Matuda 31527 & ENCB & Anomocítico & 20 & 12 & 62 \\
\hline
\end{tabular}

Se observó que en las especies estudiadas los estomas sólo están presentes en el envés de la hoja por lo que dichos taxa se clasifican como hipostomáticas (Fahn, 1978).

\section{AGRADECIMIENTOS}

Agradecemos a la Biól. Adriana Bernal Pedreira y al M. en C. Ezequiel Carlos Rojas Zenteno por la elaboración de los dibujos. A los curadores de los herbarios ENCB, IZTA y MEXU por el préstamo de los ejemplares que se revisaron. A la P. B. Gabriela Padilla por su ayuda en la elaboración de las figuras.

\section{LITERATURA CITADA}

Conover, M. 1983. The vegetative morphology of the reticulate-veined Liliiflorae. Telopea 2: 401-412.

Cuerrier, A., L. Brouillet y D. Barabé. 1990. Numerical taxonomic study of the Begoniaceae using the Mantel test on leaf microcharacters. Taxon 39: 549-560. 
Dilcher, D. 1974. Approaches to the identification of angiosperm leaf remains. Bot. Rev. 40(1): 91-103.

Fahn, A. 1978. Anatomía vegetal. H. Blume Ediciones. Madrid. 643 pp.

Foster, A. S. 1950. Morphology and leaf venation of the leaf of Quiina acutangula. Am. J. Bot. 37: 159-171.

Franck, D. H. 1976. Leaf venation in certain Betulaceae: ontogenic and ecological considerations. Am. J. Bot. 37: 297-305

Galndolfo, M. A. y E. J. Romero. 1992. Leaf morphology and a key to species of Nothofagus Bull. B. Torrey Bot. Club 119: 152-166.

Hershkovitz, M. A. 1991. Leaf morphology of Cistanthe Spach (Portulacaceae). Ann. Mo. Bot. Gard. 78: 1022-1060.

Hickey, L. J. 1973. Classification of the architecture of dicotyledonous leaves. Am. J. Bot. 60: 17-33.

Hickey, L. J. 1974. Clasificación de la arquitectura de las hojas de dicotiledóneas. Bol. Soc. Arg. Bot. 26(12): 1-26.

Hickey, L. J. y D. W. Taylor. 1989. Reexamination of leaf architectural characters of basal angiosperms and their sister-groups: implications for the origin and relationship of angiosperms. Am. J. Bot. 76(6): 245.

Hickey, L. J., J. Ash, B. Ellis, K. Jonson, P. Wilf y S. Wing. 1999. Manual of leaf architecture morphological description and categorization of dicotyledonous and net-veined monocotyledonous angiosperms. Leaf Architecture Working Group. Smithsonian Institution. Washington, D.C. 65 pp.

Jones, J. H. 1986. Evolution of the Fagaceae: The implications of foliar features. Ann. Mo. Bot. Gard. 73: 228-275.

Levin, G. 1986. Systematic foliar morphology of Phyllanthoideae (Euphorbiaceae). Cladistic analysis. Syst. Bot. 3(11): 515-530.

Lis, R. A. 1992. Leaf architectural survey of Cercocarpus (Rosaceae) and its systematic significance. Int. J. Plant Sci. 153: 258-272.

Llamas, F., C. Pérez-Morales, C. Acedo y A. Penas. 1995. Foliar trichomes of the evergreen and semi-deciduous species of the genus Quercus (Fagaceae) in the Iberian Peninsula. Bot. J. Linn. Soc. 117: 47-57.

Manos, P. 1993. Foliar trichome variation in Quercus section Protobalanus (Fagaceae). Sida 15: 391-403.

Martínez, M. 1954. Los encinos de México. Bol. Soc. Bot. Méx. 28: 29-179.

Martínez, D. 2002. Variación morfométrica de dos especies de encinos rojos: Quercus sartorii Liebmann y Quercus xalapensis Humboldt \& Bonpland (Fagaceae). Tesis de maestría. Colegio de Postgraduados. Montecillo, Edo. de México. 101 pp.

Miranda, F. y E. Hernández-X, 1963. Los tipos de vegetación de México y su clasificación. Bol. Soc. Bot. Méx. 28: 29-179.

Nixon, K. 1993. The genus Quercus in Mexico. In: Ramamoorthy, T. P., R. Bye, A. Lot y J. Fa (eds.). Biological diversity of Mexico: origins and distribution. Oxford University Press. Nueva York. pp. 447-458. 
Pole, M. 1991. A modified terminology for angiosperm leaf architecture. J. Roy. Soc. New Zeal. 21: 297-312.

Pray, T. R. 1954. Foliar venation of angiosperms. I. Mature venation of Liriodendron. Am. J. Bot. 41: 663-670.

Pray, T. R. 1959. Pattern and ontogeny of the foliar venation of Bobea elatior (Rubiaceae). Pac. J. Sci. 13: 3-13.

Ramji, M. V. 1961. Morphology and ontogeny of foliar venation of Calophyllum inophyllum. Aus. J. Bot. 15: 437-443.

Romero, S. 2000. Estudio taxonómico de la serie Acutifoliae (Quercus, Fagaceae). Tesis doctoral. Facultad de Estudios Superiores Iztacala. Universidad Nacional Autónoma de México. México, D.F. 174 pp.

Romero, S., C. Rojas y M. Aguilar. 2002. El género Quercus (Fagaceae) en el Estado de México. Ann. Mo. Bot. Gard. 89: 551-593.

Rury, P. M. 1976. Leaf venation patterns of the genus Hibbertia Andr. (Dilleniaceae). Am. J. Bot. 58: 209-241.

Rzedowski, J. 1978. Vegetación de México. Ed. Limusa. México, D.F. 432 pp.

Todzia, C. A. y R. C. Keating. 1991. Leaf architecture of the Chloranthaceae. Ann. Mo. Bot. Gard. 78: 476-496.

Trelease, W. 1924. The American oaks. Mem. Nat. Acad. Sci. 20: 1-255.

Valencia, S. y A. Delgado. 1995. Arquitectura de hojas como una herramienta taxonómica en el reconocimiento de individuos de encinos pertenecientes a Quercus affinis Scheid. y Quercus laurina Humboldt \& Bonpland. In: Marroquín de la Fuente, J. S. (ed.). Memorias del III Seminario Nacional de Encinos, tomo II; 4-6 de Noviembre de 1995. Universidad Autónoma de Nuevo León. Linares, N.L. pp. 850-859

Zavala, F. 1995. Encinos hidalguenses. Universidad Autónoma Chapingo. Chapingo, México. 133 pp. 